\title{
Light-induced spin polarizations in quantum rings
}

\author{
Fateme K. Joibari, ${ }^{1}$ Ya. M. Blanter, ${ }^{1}$ and Gerrit E. W. Bauer ${ }^{1,2}$ \\ ${ }^{1}$ Kavli Institute of NanoScience, Delft University of Technology, Delft, The Netherlands \\ ${ }^{2}$ Institute for Materials Research and WPI-AIMR, Tohoku University, Sendai, Japan
}

(Received 3 March 2014; revised manuscript received 8 September 2014; published 2 October 2014)

\begin{abstract}
Nonresonant circularly polarized electromagnetic radiation can exert torques on magnetizations by the inverse Faraday effect (IFE). Here, we discuss the enhancement of IFE by spin-orbit interactions. We illustrate the principle by studying a simple generic model system, i.e., the quasi-one-dimensional ring in the presence of linear/cubic Rashba and Dresselhaus interactions. We combine the classical IFE in electron plasmas that is known to cause persistent currents in the plane perpendicular to the direction of the propagation of light with the concept of current and spin-orbit-induced spin transfer torques. We calculate light-induced spin polarization that in ferromagnets might give rise to magnetization switching.
\end{abstract}

DOI: 10.1103/PhysRevB.90.155301

PACS number(s): 75.70.Tj, 75.75.-c, 75.60.Jk

\section{INTRODUCTION}

The Faraday effect (FE) describes the rotation of the plane of linearly polarized light when passing through a ferromagnet with a magnetization component parallel to the light vector. It is caused by the difference of phase shifts of transmitted light in the two circular polarization states. The inverse Faraday effect (IFE) is the ability of circularly polarized light to induce a spin polarization in metals and to reorient or even reverse the magnetization in ferromagnets. In other words, the IFE stands for effective circularly polarized light-induced magnetic fields in the direction of the light wave vector with sign governed by the helicity and magnitude scaling with the intensity. In contrast to other photomagnetic effects, neither FE nor IFE involve absorption of photons, which makes them potentially very fast and therefore interesting, e.g., for data storage technologies.

IFE was initially predicted by Pitaevskii [1] and formulated in terms of the dependence of the free energy on a timedependent electric field. After observation of IFE by van der Ziel et al. [2], Pershan et al. [3] developed a microscopic theory explaining IFE in terms of an optically induced splitting of degenerate spin levels, followed by thermal relaxation. They predicted that magnetization $\mathbf{M}=V \lambda_{0}(2 \pi c)^{-1}\left(I_{R}-I_{L}\right) \mathbf{e}_{k}$ was created by the circularly polarized light propagating in the $\mathbf{e}_{k}$ direction with the intensity $I_{R(L)}$ of the right- (left-) handed circularly polarized light component. Here, $V, \lambda_{0}$, and $c$ are the Verdet constant, the wavelength, and the speed of the light, respectively. Popova et al. [4,5] generalized the Pershan et al. [3] approach to include the time dependence of the light field that becomes relevant on the fs time scale, and applied the formalism to isolated atoms.

Kimel et al. demonstrated IFE in $\mathrm{DyFeO}_{3}$ by exciting magnetization dynamics with circularly polarized laser pulses on fs time scales [6]. These and subsequent experiments as reviewed in Ref. [7] are not fully explained by the theory presented by Pershan et al. [3] because thermal relaxation does not occur at such short-time scales. Subsequently, Stanciu et al. [8] demonstrated that the perpendicular magnetization of GdFeCo thin films can be switched on subpicosecond time scale. Vahaplar et al. [9] modeled the switching process by multiscale calculations of the magnetization dynamics [10] with effective magnetic fields of the order of $20 \mathrm{~T}$. However, the microscopic origin, magnitude, and material dependence of these fields remain unexplained.

The reciprocity between FE and IFE is not universally observed [11], and was found by theory to break down in the presence of absorption [12]. Taguchi et al. calculated the effect of terahertz electromagnetic radiation on disordered metals with spin-orbit interaction (SOI) [13]. They found a light-induced magnetization, but at the cost of light absorption. This is in contrast to the IFE phenomenology. Recently, strong effective magnetic fields were calculated for magnetic semiconductors that are caused by the spin-selective dynamical Stark effect [14].

IFE has also been studied in classical plasmas, where it can be explained in terms of the Ørsted magnetic fields generated by light-induced circulating dc charge currents [15-18]. Hertel investigated this process for solid-state electron plasmas [19]. He derived the eddy currents and associated magnetic fields generated by time-dependent circularly polarized light in a conducting metal film modeled as a collisionless electron gas. Both currents and the related magnetic fields are dissipationless and scale to second order in the electric field amplitude of the circularly polarized light, in line with the microscopic theories for IFE. However, these effects are too small to explain the light-induced magnetization switching. Yoshino discussed dissipative corrections to Hertel's theory [20].

Here, we pursue the concept that IFE is caused by lightinduced dc currents, but invoke the spin-orbit interaction to explain the large effective fields apparently at work. This perspective of IFE is motivated by the linear current-driven intrinsic spin torque in ferromagnets predicted by Manchon and Zhang [21,22], who demonstrated that current in the presence of SOI of the Rashba-type produces an effective magnetic field which is perpendicular to both an inversion symmetry-breaking electric field and the current. The nondissipative currents discussed above can be interpreted as a reactive response to a light field, or as a ground-state property of the system in the presence of the light field, quite analogous to persistent currents or diamagnetic response to magnetic field that can be formulated as ground states in the presence of a vector potential [23]. The quantum-mechanical ground-state nature of the light-induced current in a one-dimensional (1D) ring has been investigated by Kibis [24]. A theory of the IFE can be based on Kibis' approach but for Hamiltonians with 
spin-orbit interactions. Rather than focusing on the quantum mechanics of the generation of charge currents by the light field, we concentrate here on the generation of effective magnetic fields in the presence of circulating charge currents, while using Hertel's approach to estimate the magnitude of these currents for a given light intensity. This is allowed in the high-frequency limit in which the length scale associated with the direct response is much smaller than the geometric confinement or the spin-orbit precession length.

In order to establish the principle, we focus here on a nonmagnetic system with spin-orbit interaction and in the presence of electron current bias that is generated by circularly polarized light. For a magnetic sample, such polarization can exert spin-orbit torques on magnetization. We focus on a simple yet realistic model system in which the spin-orbit interaction Hamiltonian is well known and analytical results can be achieved, viz., a one-dimensional (1D/single transverse mode) ring fabricated from a high-mobility two-dimensional electron/hole gas (2DEG/2DHG) with Rashba and Dresselhaus SOI interactions. SOI in a $2 \mathrm{DEG}$ that is linear in the wave vector is known to be quite anomalous, causing, e.g., vanishing spin Hall effect by impurity scattering [25]. Here, we find that light-induced effective fields in 1D rings with linear Rashba or Dresselhaus SOI also vanish, which can be traced to the state independence of the equilibrium spin texture. The holes of a 2DHG close to the valence band edge can also be described by Rashba and Dresselhaus SOI interactions, but with a cubic dependence on the wave vectors [26,27]. A quantum ring containing a hole gas has an out-of-plane state-dependent spin texture that indeed generates the current-induced spin polarization. In a ferromagnet, these would indeed induce torques on a magnetization, thereby confirming our working hypothesis.

The remainder of the paper is organized as follows. We solve the problem of a ground state in the presence of a given charge current by the method of Lagrange multipliers as explained in Sec. II. In Sec. III, we apply this method to a simple case of rings in the absence of SOI, and discuss the difference of the ground-state current induced by Lagrange multiplier and the one induced by the magnetic field in a ring. In Sec. IV, we discuss different models of SOI in more detail. In Secs. IV A and IV B, we address rings consisting of electrons in the presence of linear Rashba or Dresselhaus SOI, respectively, in which the current-induced spin polarization vanishes. In Secs. IV C and IV D, we continue with a $p$-doped quantum ring, in which a current-induced polarization is generated by the cubic Dresselhaus or Rashba SOI, respectively. We summarize our conclusions in Sec. V.

\section{METHOD OF LAGRANGE MULTIPLIERS}

We are interested in the ground state of a conductor in the presence of currents induced by an external perturbation such as electric field of light. Rather than diagonalizing the Hamiltonian in the presence of electric field [24], we calculate the ground state for given persistent current. Hereby, we lose some subtle nonperturbative quantum effects [24] beyond the current generation, which can be important when microwave frequencies are tuned to lie between quantized states. This is beyond the scope of this paper.
According to the current-density-functional theory [28], the ground-state energy of a system is a functional of the charge current distribution $\mathbf{j}_{\text {ext }}(\mathbf{r})$. The minimum energy of the system under the constraint of given $\mathbf{j}_{\text {ext }}(\mathbf{r})$ can be found by the method of Lagrange multipliers. Here, the Hamiltonian $H_{0}$ is augmented by the sum of the product of constraints and Lagrange multipliers that in continuous systems becomes an integral. We limit attention to noninteracting systems with single-particle states $\left|\Psi_{i}\right\rangle$ and occupation numbers $f_{i} \in\{0,1\}$ with $\sum_{i=1}^{\infty} f_{i}=N$ for a number of $N$ electrons. We may then express the constraint as

$$
\sum_{i} f_{i} \mathbf{j}_{i}(\mathbf{R})=\mathbf{j}_{\mathrm{ext}}(\mathbf{R})
$$

where the current operator $\hat{\jmath}(\mathbf{R})$ is defined in terms of the expectation value

$$
\begin{aligned}
\mathbf{j}_{i}(\mathbf{R}) & =\left\langle\Psi_{i}|\hat{\jmath}(\mathbf{R})| \Psi_{i}\right\rangle \\
& =\frac{e}{2} \int \Psi_{i}^{*}(\mathbf{r})[\mathbf{v} \delta(\mathbf{r}-\mathbf{R})+\delta(\mathbf{r}-\mathbf{R}) \mathbf{v}] \Psi_{i}(\mathbf{r}) d \mathbf{r} \\
& =e \operatorname{Re} \Psi_{i}^{*}(\mathbf{R}) \mathbf{v} \Psi_{i}(\mathbf{R}) \neq e\left\langle\Psi_{i}|\mathbf{v}| \Psi_{i}\right\rangle
\end{aligned}
$$

and $\mathbf{v}$ is the velocity operator. The objective functional under this constraint and the normalization condition $\left\langle\Psi_{i} \mid \Psi_{i}\right\rangle=1$ is

$$
\begin{aligned}
F\left[\left\{\Psi_{i}\right\}, \mathbf{j}_{\text {ext }}\right]= & \sum_{i} f_{i}\left[\left\langle\Psi_{i}\left|H_{0}\right| \Psi_{i}\right\rangle-\varepsilon_{i}\left(\left\langle\Psi_{i} \mid \Psi_{i}\right\rangle-1\right)\right] \\
& +\int \mathbf{A}(\mathbf{R}) \cdot\left(\mathbf{j}_{\text {ext }}(\mathbf{R})-\sum_{i} f_{i} \mathbf{j}_{i}(\mathbf{R})\right) d \mathbf{R} .
\end{aligned}
$$

Here, $\mathbf{A}$ is the Lagrange multiplier functional. Minimizing $F$, i.e., $\delta F / \delta \Psi_{i}^{*}=0$, leads to the Schrödinger equation with the eigenfunctions $\left|\Psi_{i}\right\rangle$ corresponding to the Hamiltonian

$$
H=H_{0}-\int \mathbf{A}(\mathbf{R}) \cdot \hat{\jmath}(\mathbf{R}) d \mathbf{R} .
$$

In the absence of spin-orbit interactions $\mathbf{j}_{i}=$ $(e \hbar / m) \operatorname{Im} \Psi_{i}^{*} \nabla \Psi_{i}$ and

$$
H(\mathbf{r}, \mathbf{p}) \rightarrow H_{0}[\mathbf{r}, \mathbf{p}-e \mathbf{A}(\mathbf{r})]-\frac{\hbar^{2} e^{2} \mathbf{A}^{2}(\mathbf{r})}{2 m} .
$$

When the objective current density $\mathbf{j}_{\text {ext }}(\mathbf{r})$ is constant in space and time, the Lagrange function $\mathbf{A}(\mathbf{r})$ is a vector potential corresponding to constant magnetic field, and the implementation of the charge current constraint is equivalent to a gauge transformation. We note the close relation with the current density functional theory [28], in which effective vector and scalar potentials are introduced to construct energy functionals of charge and current densities. Finally, we observe that the time derivative of the vector potential is the electric field $\mathbf{E}=$ $-d \mathbf{A} / d t$. Harmonic ac electric field therefore corresponds to a vector potential in the same direction with the amplitude $A_{\omega}=-i E_{\omega} / \omega$ in frequency space. The effect of finite $A_{\omega}$ in the dc limit $\omega \rightarrow 0$ is then equivalent to the transport response to electric field that remains finite in a ballistic system. Alternatively, we can associate the vector potential with applied magnetic field inducing persistent ground-state current, although it should be kept in mind that when the 
current is generated by other means, our magnetic field is a fictitious one.

\section{SINGLE-MODE QUANTUM RINGS WITHOUT SOI}

In the following, we focus on quantum rings fabricated from $2 \mathrm{DE}(\mathrm{H}) \mathrm{G}$ in which the charge carriers are confined normal to the plane by a potential $V(z)$ and in the radial direction by an axially symmetric confining potential $U(r)$ centered at an effective radius $r=a$, but free to move along the azimuthal direction along the unit vector $\mathbf{e}_{\varphi}$. In the envelope function approximation with effective mass $m$ for electrons or (heavy) holes

$$
H_{0}=\frac{p_{x}^{2}+p_{y}^{2}}{2 m}+V(z)+U(r),
$$

where $p_{x(y)}$ is the $x(y)$ component of the momentum operator. The eigenstates are then separable as $\Psi_{n l k}(r, \varphi, z)=$ $\psi_{n}(\varphi) R_{l}(r) Z_{k}(z) \quad$ normalized as $\int\left|\psi_{n}(\varphi)\right|^{2} d \varphi=$ $\int r\left|R_{l}(r)\right|^{2} d r=\int\left|Z_{k}(z)\right|^{2} d z=1$. To simplify the problem further, we assume that the confinement is strong enough such that only the lowest subbands $(k=l=0)$ are occupied, which makes the system effectively one dimensional (1D) in azimuthal direction. The eigenstates of Eq. (8) are

$$
\psi_{n}(\varphi)=\frac{1}{\sqrt{2 \pi}} e^{i n \varphi}
$$

with the energies $\varepsilon_{n}=\hbar^{2} n^{2} /\left(2 m a^{2}\right)+\varepsilon_{0}$, where $\varepsilon_{0}$ is the confinement energy corresponding to $R_{0}(r) Z_{0}(z)$.

We wish to model the system in the presence of constant persistent current. In the absence of SOI, the current operator along the ring is defined by its expectation value

$$
\begin{aligned}
\mathbf{j}_{n}^{\varphi}(r, z) & =j_{n}^{\varphi}(r, z) \mathbf{e}_{\varphi} \\
& =\mathbf{e}_{\varphi} \frac{e \hbar}{m r}\left|R_{0}(r)\right|^{2}\left|Z_{0}(z)\right|^{2} \operatorname{Im} \psi_{n}(\varphi) \frac{\partial}{\partial \varphi} \psi_{n}(\varphi),
\end{aligned}
$$

where we used $v_{\varphi}=-i \hbar /(m r) \partial / \partial \varphi$, and the total current in the wire is

$$
\begin{aligned}
I^{\varphi} & =\iint d z d r j^{\varphi}(r, z) \\
& =-\frac{e \hbar}{m} \operatorname{Im} \psi_{n}(\varphi) \frac{\partial}{\partial \varphi} \psi_{n}(\varphi) \int d r \frac{1}{r}\left|R_{0}(r)\right|^{2} \int d z\left|Z_{0}(z)\right|^{2} \\
& =-\frac{e \hbar}{m a^{2}} \sum_{n} f_{n} \operatorname{Im} \psi_{n}(\varphi) \frac{\partial}{\partial \varphi} \psi_{n}(\varphi),
\end{aligned}
$$

where $e>0$, and we used $\int d r\left|R_{0}(r)\right|^{2} / r=1 / a^{2}$ assuming Gaussian $R_{0}$ [29]. The current operator (3) is diagonal in the basis of the states (9), which are therefore also eigenfunctions of the current carrying system. The total current density in the ring then reads as

$$
I^{\varphi}=-\frac{e \hbar}{2 \pi m a^{2}} \sum_{n} f_{n} n .
$$

The projected Hamiltonian $\left\langle Z_{0} R_{0}\left|H_{0}\right| Z_{0} R_{0}\right\rangle$ in the presence of the Lagrange multiplier term $-A^{\varphi} \hat{I}^{\varphi}$ (parametrizing the vector potential as $A^{\varphi}=2 \pi \hbar n_{\lambda} / e$ where $n_{\lambda}$ is dimensionless) is diagonal in the basis (9) with the energies

$$
\begin{aligned}
\varepsilon_{n} & =E_{a} n^{2}-\frac{2 \pi \hbar n_{\lambda}}{e} \frac{e \hbar}{2 \pi m a^{2}} n+\varepsilon_{0} \\
& =E_{a}\left(n-n_{\lambda}\right)^{2}+\tilde{\varepsilon}_{0},
\end{aligned}
$$

where $E_{a}=\hbar^{2} /\left(2 m a^{2}\right)$ and $\tilde{\varepsilon}_{0}=\varepsilon_{0}-E_{a} n_{\lambda}^{2}$. At zero temperature $f_{n}=\Theta\left(\varepsilon_{n}-\epsilon_{F}+\tilde{\varepsilon}_{0}\right)$, where $\epsilon_{F}$ is the Fermi energy and $\Theta$ the step function, therefore,

$$
I^{\varphi} \approx \frac{2 e \hbar}{\pi m a^{2}} n_{\lambda} n_{F},
$$

where $n_{F}=\sqrt{\left(\epsilon_{F}-\tilde{\varepsilon}_{0}\right) / E_{a}}$. We assume that the number of electrons is constant under variation of $n_{\lambda}$, which implies that $\tilde{\varepsilon}_{0}$ may be set to zero. The current constraint $I^{\varphi}=I$ determines the effective vector potential

$$
n_{\lambda}=\frac{\pi m a^{2}}{2 e \hbar n_{F}} I=\frac{\pi}{4 e} \frac{\hbar}{E_{a}} \frac{I}{n_{F}}
$$

so that the spectrum (16) is fully determined. The current is optimally accommodated by rigidly shifting the distribution function proportional to the applied current.

Real magnetic field $B_{\text {ext }}$ also generates persistent currents [23]. There is a difference, however. The energies of a quantum ring in the presence of a real magnetic flux $\Phi=\pi a^{2} B_{\text {ext }}$ read as

$$
E_{n}=E_{a}\left(n-\frac{\Phi}{\Phi_{0}}\right)^{2}
$$

where $\Phi_{0}=e / h$ is the flux quantum and we can identify $n_{\lambda}=\Phi / \Phi_{0}$. The total energy in the presence of diamagnetic persistent current is

$$
E^{\prime}=\sum_{n_{F}^{(-)}}^{n_{F}^{(+)}} E_{n}
$$

where $n_{F}^{( \pm)}=\left\lfloor \pm \sqrt{2 m a^{2}\left(\epsilon_{F}-\varepsilon_{0}\right)} / \hbar+\Phi / \Phi_{0}\right\rfloor$ is the largest integer smaller of equal $\sqrt{2 m a^{2}\left(\epsilon_{F}-\varepsilon_{0}\right)} / \hbar$. $E^{\prime}(\Phi)$ is periodic since the quantum numbers of the highest occupied states jump by \pm 1 when two states cross the Fermi energy. The current

$$
I^{\varphi \prime}=\frac{\partial}{\partial \Phi} E^{\prime}=-\Phi_{0} E_{a} \sum_{n_{F}^{(-)}}^{n_{F}^{(+)}}\left(n-\frac{\Phi}{\Phi_{0}}\right)
$$

oscillates as a function of $\Phi$ with the maximum

$$
\left|I^{\varphi \prime}\right|_{\max }=N E_{a} \Phi_{0}=1.5 \times 10^{-10} \mathrm{~A} \frac{N}{1000} \frac{0.1 \mu \mathrm{m}}{a^{2}},
$$

where $N=2\left(n_{F}^{(+)}+n_{F}^{(-)}\right)$is the total number of electrons.

The Lagrange multiplier on the other hand contributes the additional term $\hbar^{2} n_{\lambda}^{2} /\left(2 m a^{2}\right)$ [see Eq. (16)], which modifies the expressions to

$$
\begin{gathered}
\frac{E_{n}}{E_{a}}=\left(n-n_{\lambda}\right)^{2}-n_{\lambda}^{2}=n\left(n-2 n_{\lambda}\right), \\
I^{\varphi}=\Phi_{0} \frac{\partial E}{\partial n_{\lambda}}=-E_{a} \Phi_{0} \sum_{-n_{F}-n_{\lambda}}^{n_{F}-n_{\lambda}} n,
\end{gathered}
$$

which agrees with Eq. (15).

The current is finite for any $n_{\lambda} \neq 0$ (except when $N=$ 1 and $n=0$ or $2 n_{\lambda}$ ). Thus, contrary to the diamagnetic current induced by real magnetic field, the Lagrangian method 


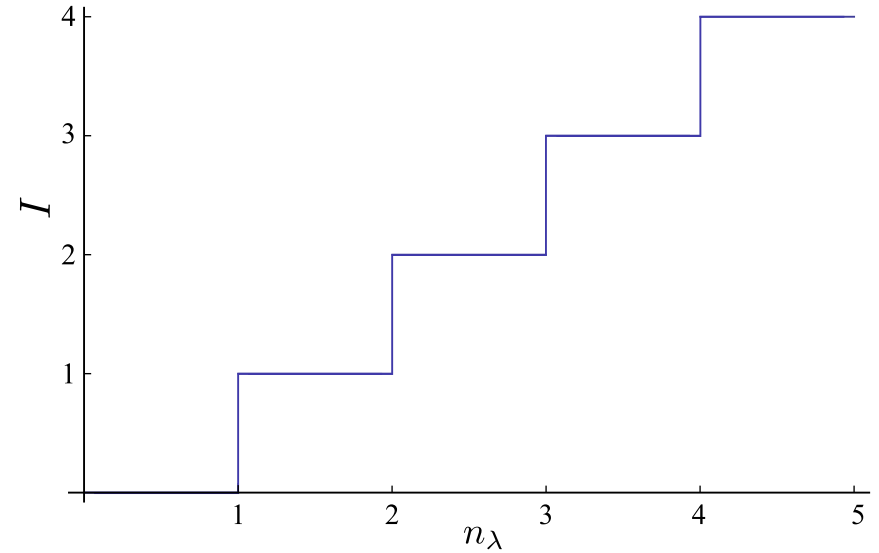

FIG. 1. (Color online) Current versus $n_{\lambda}$. The current axis is scaled in units of $I_{0}=2 e \hbar n_{F} /\left(\pi m a^{2}\right)$.

generates unbound currents. However, due to the discreteness of the energy levels, the currents are quantized (see Fig. 1). In the following, we work with a large number of electrons such that the currents are quasicontinuous.

Nevertheless, if it is taken into account that there is a maximum for magnetic-field-induced currents, our method also predicts spin polarization generated by diamagnetic currents and correspondingly enhanced paramagnetic susceptibility of quantum rings. It is instructive to compare the magnitudes of the Lagrange multipliers with the corresponding magnetic fields. With $e A^{\varphi}=2 \pi n_{\lambda}=e B_{\mathrm{ext}} a^{2} /(2 \hbar)$,

$$
\begin{aligned}
B_{\text {ext }} & =\left(\frac{\pi \hbar}{e a}\right)^{2} \frac{1}{E_{a}} \frac{I}{n_{0}}=\left(\frac{\pi}{e}\right)^{2} \frac{2 m}{n_{0}} I \\
& \approx 0.1 \mathrm{mT} \frac{1000}{n_{0}} \frac{I}{\mathrm{nA}},
\end{aligned}
$$

which does not depend on the size of the ring.

The generation of charge current by circularly polarized light is discussed in Appendix following Hertel [19]. We find that for experimentally accessible light intensities, $B_{\text {ext }}$ can be of the order of $1 \mathrm{~T}$.

\section{SINGLE-MODE RINGS IN THE PRESENCE OF SOI}

In the weakly relativistic limit, a particle spin experiences SOI, i.e., effective magnetic field that scales with the particle velocity. It requires inversion symmetry breaking induced either by space charges or asymmetric heterostructures or by a unit cell without inversion symmetry, as is the case for the zinc-blende structure. The Rashba SOI in quasi-twodimensional electron gas (2DEG) is a simple realization of the former [30,31] while the Dresselhaus [32] SOI represents the latter type. For 2DEG in the $x, y$ plane, the Hamiltonian (8) is then augmented by

$$
H_{\mathrm{SO}}^{e}=\frac{\alpha_{e}}{\hbar}\left(\sigma_{y} p_{x}-\sigma_{x} p_{y}\right)+\frac{\beta_{e}}{\hbar}\left(\sigma_{x} p_{x}-\sigma_{y} p_{y}\right),
$$

where $\sigma_{x(y)}$ are the $x(y)$ components of the momentum operator for electrons and a vector of the Pauli matrices, respectively. In two-dimensional hole gas (2DHG), on the other hand $[27,33,34]$,

$$
H_{\mathrm{SO}}^{h}=\left(i \frac{\alpha_{h}}{\hbar^{3}} p_{-}^{3}+\frac{\beta_{h}}{\hbar^{3}} p_{-} p_{+} p_{-}\right) \sigma_{+}+\text {H.c. },
$$

and $O_{ \pm}=O_{x} \pm i O_{y}$, where $\mathbf{O} \equiv \mathbf{p}, \boldsymbol{\sigma}$, are the momentum operator and the Pauli spin matrix vectors, respectively. $\alpha_{e(h)}$ and $\beta_{e(h)}$ parametrize the linear (cubic) Rashba and the linear (cubic) Dresselhaus SOI. The canonical velocity operators are modified by the spin-orbit interaction since they do not commute with the Hamiltonian. Dropping the index for electrons and holes,

$$
\begin{aligned}
\mathbf{v} & =\dot{\mathbf{r}}=\frac{1}{i \hbar}[\mathbf{r}, H] \\
& =\mathbf{v}_{0}+\mathbf{v}_{\mathrm{SO}}=\frac{\hbar}{i m} \nabla+\frac{1}{i \hbar}\left[\mathbf{r}, H_{\mathrm{SO}}\right],
\end{aligned}
$$

where $\mathbf{v}_{\mathrm{SO}}$ is the anomalous velocity. The current operators are modified analogously.

As before, we add an axially symmetric confinement potential to the $2 \mathrm{DE}(\mathrm{H}) \mathrm{G}$ and consider the electric quantum confinement (1D) limit. Here, we separately discuss electrons and holes in such quantum rings in the presence of a circular current, and calculate the current-induced spin polarization in each system.

\section{A. Electrons with the Rashba SOI}

For electrons in the 1D quantum ring, the projection of the full Hamiltonian $H$ onto the azimuthal subspace leads to [29]

$$
\begin{aligned}
H(\varphi)= & \left\langle Z_{0} R_{0}\left|H_{0}+H_{S O}\right| Z_{0} R_{0}\right\rangle=-\frac{\hbar^{2}}{2 m a^{2}} \partial_{\varphi}^{2}-i \frac{\alpha}{a}\left\{\left(\sigma_{x} \cos \varphi+\sigma_{y} \sin \varphi\right) \partial_{\varphi}+\frac{1}{2}\left(\sigma_{y} \cos \varphi-\sigma_{x} \sin \varphi\right)\right\} \\
& -i \frac{\beta}{a}\left\{\left(\sigma_{x} \sin \varphi+\sigma_{y} \cos \varphi\right) \partial_{\varphi}+\frac{1}{2}\left(\sigma_{x} \cos \varphi-\sigma_{y} \sin \varphi\right)\right\}
\end{aligned}
$$

Let us first focus on the Rashba spin-orbit interaction, i.e., $\beta=0$. The eigenstates of the system are

$$
\begin{aligned}
& \psi_{n+}^{R}(\varphi)=\frac{1}{\sqrt{2 \pi}} e^{i n \varphi}\left(\begin{array}{c}
\cos \frac{\theta_{R}}{2} \\
\sin \frac{\theta_{R}}{2} e^{i \varphi}
\end{array}\right) ; \\
& \psi_{n-}^{R}(\varphi)=\frac{1}{\sqrt{2 \pi}} e^{i n \varphi}\left(\begin{array}{c}
-\sin \frac{\theta_{R}}{2} \\
\cos \frac{\theta_{R}}{2} e^{i \varphi}
\end{array}\right),
\end{aligned}
$$

where $n$ is an integer, with the energies

$$
\frac{E_{n \sigma}}{E_{a}}=\left(n+\frac{1}{2}\right)^{2}+\sigma\left(n+\frac{1}{2}\right) \sec \theta_{R}+\frac{1}{4},
$$

where $\tan \theta_{R}=2 m a \alpha / \hbar^{2}$. The velocity operator in this system reads as

$$
v_{\varphi}=-\frac{i \hbar}{m a} \partial_{\varphi}+\frac{\alpha}{\hbar} \sigma_{r}
$$


and the current is $\left\langle I^{\varphi}\right\rangle=\sum_{n \sigma} f_{n \sigma} I_{n \sigma}^{\varphi}=I$. The current operator is diagonal in the $n \sigma$ basis [Eqs. (33) and (34)], but acquires a spin dependence

$$
\begin{aligned}
I_{n \sigma}^{\varphi}= & -\frac{e \hbar}{2 \pi m a^{2}} n-\sigma \frac{e \alpha}{2 \pi \hbar a} \sin \theta \\
& -\frac{e \hbar}{2 \pi m a^{2}}\left(\delta_{\sigma,+1} \cos ^{2} \frac{\theta}{2}+\delta_{\sigma,-1} \sin ^{2} \frac{\theta}{2}\right) .
\end{aligned}
$$

The projected Hamiltonian in the presence of the Lagrange multiplier term (parametrizing the vector potential as $A^{\varphi}=$ $\hbar n_{\lambda} / e$ where $n_{\lambda}$ is dimensionless) is diagonal in the basis (9) with the energies

$$
\begin{aligned}
\frac{E_{n \sigma}}{E_{a}}= & \left(n+\frac{1}{2}\right)^{2}+\sigma(n+1 / 2) \sec \theta_{R}+\frac{1}{4} \\
& -\frac{2 \pi \hbar n_{\lambda}}{e} \frac{2 m a^{2}}{\hbar^{2}}\left[\frac{e \hbar}{2 \pi m a^{2}} n+\sigma \frac{e \alpha}{2 \pi \hbar a} \sin \theta_{R}\right. \\
& \left.+\frac{e \hbar}{2 \pi m a^{2}}\left(\delta_{\sigma,+1} \cos ^{2} \frac{\theta_{R}}{2}+\delta_{\sigma,-1} \sin ^{2} \frac{\theta_{R}}{2}\right)\right] \\
= & \left(n-n_{\lambda}+\frac{1}{2}\right)^{2}+\sigma\left(n-n_{\lambda}+\frac{1}{2}\right) \sec \theta_{R}+\frac{1}{4}-n_{\lambda}^{2} .
\end{aligned}
$$

At zero temperature,

$$
I=\sum_{n \sigma} f_{n \sigma} I_{n \sigma}^{\varphi}=\sum_{\sigma} \sum_{-n_{r}+n_{\lambda \lambda}-\sigma \sec \theta_{R}-1 / 2}^{n_{r}+n_{\lambda \lambda}-\sigma \sec \theta_{R}-1 / 2} I_{n \sigma}^{\varphi}=\frac{2 e \hbar}{\pi m a^{2}} n_{\lambda} n_{r},
$$

where $n_{r} \equiv \sqrt{\epsilon_{F} / E_{a}+\sec ^{2} \theta_{R} / 4}$ and we substituted Eq. (37). The leading term is therefore the same as in the absence of spin-orbit interaction:

$$
n_{\lambda}=\frac{\pi}{4 e} \frac{\hbar}{E_{a}} \frac{I}{n_{r}}
$$

Since the system is not magnetic, it is not spin polarized at equilibrium. The spin polarization of the current-carrying ground state reads as

$$
\left\langle\sigma_{z}\right\rangle_{I}^{R}=\sum_{n \sigma}\left\langle\psi_{n \sigma}^{R}\left|\sigma_{z}\right| \psi_{n \sigma}^{R}\right\rangle_{I}=\sum_{n \sigma} f_{n \sigma} \sigma \cos \theta_{R}
$$

and $\left\langle\sigma_{y}\right\rangle_{I}^{R}=\left\langle\sigma_{x}\right\rangle_{I}^{R}=0$. In the absence of current, the energy bands are equally filled for both spins in the negative and positive directions, and we do not have unpaired electrons.
Thus,

$$
\begin{aligned}
\left\langle\sigma_{z}\right\rangle_{I=0}^{R} & =\cos \theta_{R} \sum_{n \sigma} \sigma \Theta\left(\epsilon_{F}-E_{n \sigma}\right) \\
& =\cos \theta_{R} \sum_{\sigma} \sum_{n_{n}+n_{\lambda}-\sigma \sec \theta_{R}-1 / 2}^{n_{r}+n_{\lambda}-\sigma \sec \theta_{R}-1 / 2} \sigma=0 .
\end{aligned}
$$

In the presence of the current bias, the electron distribution is shifted in reciprocal space around the Fermi level by $n_{\lambda}$. The spinors (33) and (34) that determine the spin texture do not depend on $n$. Furthermore, the relative occupation of the two spin bands also remains the same. Therefore, the induced current does not generate spin polarization and $\left\langle\sigma_{z}\right\rangle_{I}^{R}=0$ for all current levels. To put it differently, since the ring is invariant to rotation, the system is invariant to a Galilean gauge transformation that induces the persistent current. The conclusion that there is no current-induced spin accumulation in the Rashba systems holds also for 1D wires. Vanishing of the spin accumulation is caused by the compensating effect of the two subbands. This can be suppressed when a gap is induced at $k=0$ by a Zeeman field or exchange interfaction and the Fermi energy is tuned to fall into this gap [35]. We also note that the linear current-induced spin accumulation does not vanish in two-dimensional electron gas either [36].

\section{B. Electrons with the Dresselhaus SOI}

A similar situation arises for a ring with only linear Dresselhaus interaction, i.e., $\alpha=0$ in Eq. (32). Its eigenstates are [37]

$$
\begin{array}{cc}
\psi_{n+}^{D}(\varphi)= & \frac{1}{\sqrt{2 \pi}} e^{i n \varphi}\left(\begin{array}{c}
-\sin \frac{\theta_{D}}{2} \\
i \cos \frac{\theta_{D}}{2} e^{-i \varphi}
\end{array}\right) ; \\
\psi_{n-}^{D}(\varphi)= & \frac{1}{\sqrt{2 \pi}} e^{i n \varphi}\left(\begin{array}{c}
\cos \frac{\theta_{D}}{2} \\
i \sin \frac{\theta_{D}}{2} e^{-i \varphi}
\end{array}\right),
\end{array}
$$

with the energies identical to those for the Rashba ring

$$
\frac{E_{n \sigma}}{E_{a}}=\left(n+\frac{1}{2}\right)^{2}+\sigma\left(n+\frac{1}{2}\right) \sec \theta_{D}+\frac{1}{4},
$$

but now $\tan \theta_{D}=2 m a \beta / \hbar^{2}$. Thus, the spin texture does not depend on the angular momentum. This means that shifting a distribution function rigidly does not change the balance of the spin states and, as in the Rashba case, there is no currentinduced spin polarization.

\section{Holes with the Dresselhaus SOI}

Stepanenko et al. [38] derived an effective low-energy Hamiltonian for heavy holes from the Luttinger Hamiltonian that includes Dresselhaus and Rashba-type SOI that are cubic in the angular momenta. Simple analytical solutions were obtained in two limits, representing the Dresselhaus-only interaction $\left(\alpha_{h}=0\right)$ and the Rashba-only SOI $\left(\beta_{h}=0\right)$. The spin textures in these two limits are shown in Fig. 2. In contrast to the electron case, both spinors include terms quadratic in the angular momentum. Here, we show that these do generate current-induced spin accumulation. 

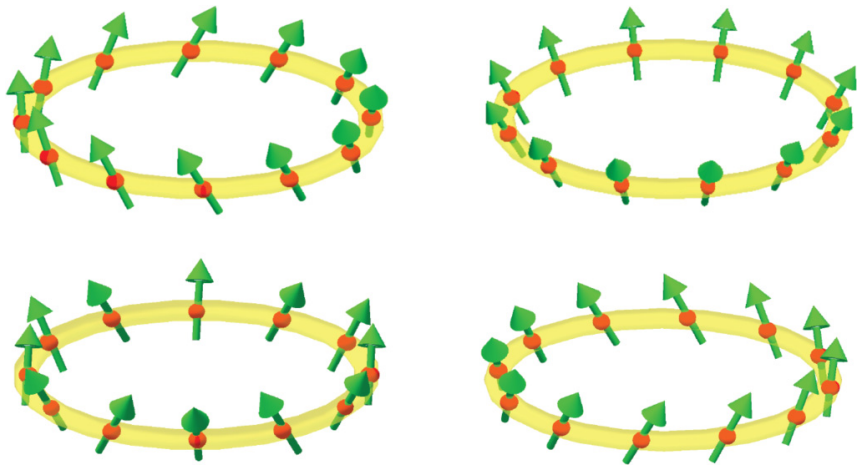

FIG. 2. (Color online) Top: electrons spin texture in the presence of the linear Dresselhaus (left) or Rashba (right) interaction. The angle of the spin with the ring in this case only depends on the SO coupling strength and not the angular momentum. Bottom: the hole spin texture in the presence of the cubic Dresselhaus (left) or Rashba (right) spin-orbit interaction. The direction of the spins in both cases depends on the angular velocity of the holes and on the SO coupling strength. Here, the particles orbit counterclockwise. The clockwise movement induces spin texture which is mirrored with respect to the plane containing the ring.

The heavy-hole Hamiltonian for a 1D ring with the Dresselhaus SOI is [38]

$$
\begin{aligned}
H_{0}^{c D}= & -\frac{\hbar^{2}}{2 m_{h h} a^{2}} \partial_{\varphi}^{2} \\
& +\beta_{h} e^{i \varphi}\left(G_{0}+G_{1} \partial_{\varphi}+G_{2} \partial_{\varphi}^{2}+G_{3} \partial_{\varphi}^{3}\right) \sigma_{-} \\
& +\beta_{h} e^{-i \varphi}\left(G_{0}-G_{1} \partial_{\varphi}+G_{2} \partial_{\varphi}^{2}-G_{3} \partial_{\varphi}^{3}\right) \sigma_{+},
\end{aligned}
$$

where $G_{0}=i\left(R_{0}+R_{1}-R_{2}\right), \quad G_{1}=-\left(R_{1}+R_{2}\right), \quad G_{2}=$ $i\left(R_{2}-2 R_{3}\right)$, and $G_{3}=-R_{3}$, and the coefficients $R_{j}=$ $\left\langle r^{-j} \partial_{r}^{3-j}\right\rangle_{\text {radial }}$ depend on the ground-state radial confinement wave function. For a ring with radius $a$ and width $w, R_{2}=$ $R_{3} / 2=1 /\left(2 a^{3}\right)$ and $R_{1}=-2 / 3 R_{0}=-1 /\left(a w^{2}\right)$ [39]. Here, $m_{h h}=m_{0} /\left(\gamma_{1}+\tilde{\gamma}\right)$, where $\tilde{\gamma}=\gamma_{2}$ for the [001] $\left(\tilde{\gamma}=\gamma_{3}\right.$ for [111]) growth direction and $\gamma_{i}$ are the standard Luttinger parameters for the valence band of III-V semiconductors. The eigenfunctions of the system are

$$
\begin{aligned}
& \psi_{l,+}^{c D}=\frac{1}{\sqrt{2 \pi}} e^{i l \varphi}\left(\begin{array}{c}
i \cos \frac{\theta^{c D}(l)}{2} e^{-i \varphi / 2} \\
-\sin \frac{\theta^{c D}(l)}{2} e^{i \varphi / 2}
\end{array}\right) ; \\
& \psi_{l,-}^{c D}=\frac{1}{\sqrt{2 \pi}} e^{i l \varphi}\left(\begin{array}{c}
i \sin \frac{\theta^{c D}(l)}{2} e^{-i \varphi / 2} \\
\cos \frac{\theta^{c D}(l)}{2} e^{i \varphi / 2}
\end{array}\right),
\end{aligned}
$$

where $l=n+1 / 2$, and the texture angle $\theta^{c D}(l)$ is

$$
\theta^{c D}(l)=\tan ^{-1}\left\{\frac{2 m_{h h} \beta_{h}}{\hbar^{2} R_{3}^{2 / 3}}\left[\frac{2}{3} R_{0}+\left(l^{2}-\frac{5}{4}\right) R_{3}\right]\right\},
$$

with the energies

$$
E_{l \sigma}=E_{a}^{h}\left(l^{2}+\frac{1}{4}+\sigma l \sec \theta^{c D}(l)\right),
$$

where $E_{a}^{h}=\hbar^{2} /\left(2 m_{h h} a^{2}\right)$. In terms of the velocity operator

$$
\begin{aligned}
v_{\varphi}= & -\frac{i \hbar}{m_{h h} a} \partial_{\varphi}+\frac{i a \beta_{h}}{\hbar} e^{i \varphi}\left(G_{1}+2 G_{2} \partial_{\varphi}+3 G_{3} \partial_{\varphi}^{2}\right) \sigma_{-} \\
& +\frac{i a \beta_{h}}{\hbar} e^{-i \varphi}\left(-G_{1}+2 G_{2} \partial_{\varphi}-3 G_{3} \partial_{\varphi}^{2}\right) \sigma_{+},
\end{aligned}
$$

the current operator reads as

$$
\hat{I}_{l \sigma \sigma^{\prime}}^{\varphi}=\frac{e}{a} \operatorname{Re} \psi_{l \sigma}^{\dagger}(\varphi) v_{\varphi} \psi_{l \sigma^{\prime}}(\varphi)
$$

Both the Hamiltonian and current operators are diagonal in the orbital angular momentum, which allows us to introduce $2 \times 2$ operators in spin space for calculation of the expectation values in position space:

$$
\begin{aligned}
-\frac{\hat{I}_{l}^{\varphi}}{e}= & 2 E_{a}^{h}\left(l_{-} \sigma_{1}+l_{+} \sigma_{2}\right) \\
& +\frac{\beta_{h}}{\hbar}\left(G_{1}+2 i G_{2} l_{-}+3 G_{3} l_{-}^{2}\right) \sigma_{-} \\
& +\frac{\beta_{h}}{\hbar}\left(G_{1}-2 i G_{2} l_{+}+3 G_{3} l_{+}^{2}\right) \sigma_{+},
\end{aligned}
$$

where $\sigma_{1}$ and $\sigma_{2}$ are $2 \times 2$ matrices with all elements zero except for the first and second diagonal one, respectively, and $l_{ \pm}=l \pm 1 / 2$. Thus, the Hamiltonian $H^{c D}+\lambda\left\langle I^{\varphi}\right\rangle$ in spin space reads as

$$
\begin{aligned}
H_{l}^{c D} & =E_{a}^{h}\left[\left(l_{-}^{2}-n_{\lambda} l_{-}\right) \sigma_{1}+\left(l_{+}^{2}-n_{\lambda} l^{+}\right) \sigma_{2}\right] \\
& -\beta_{h}\left(G_{0}+G_{1} l_{-}+G_{2} l_{-}^{2}+G_{3} l_{-}^{3}\right) \sigma_{-} \\
& -\beta_{h}\left(G_{0}-G_{1} l_{+}+G_{2} l_{+}^{2}-G_{3} l_{+}^{3}\right) \sigma_{+} \\
& +n_{\lambda} \beta_{h}\left(G_{1}+2 G_{2} l_{-}+3 G_{3} l_{-}^{2}\right) \sigma_{-} \\
& +n_{\lambda} \beta_{h}\left(-G_{1}+2 G_{2} l_{+}-3 G_{3} l_{+}^{2}\right) \sigma_{+} .
\end{aligned}
$$

The eigenstates in the presence of a current now read as

$$
\begin{gathered}
\psi_{\left(l, n_{\lambda}\right),+}^{c D}=\frac{1}{\sqrt{2 \pi}} e^{i l \varphi}\left(\begin{array}{c}
\cos \frac{\theta^{c D}\left(l, n_{\lambda}\right)}{2} e^{-(i / 2)(\varphi+\pi / 2)} \\
\sin \frac{\theta^{c D}\left(l, n_{\lambda}\right)}{2} e^{(i / 2)(\varphi+\pi / 2)}
\end{array}\right) \\
\psi_{\left(l, n_{\lambda}\right),-}^{c D}=\frac{1}{\sqrt{2 \pi}} e^{i l \varphi}\left(\begin{array}{c}
-\sin \frac{\theta^{c D}\left(l, n_{\lambda}\right)}{2} e^{-(i / 2)(\varphi+\pi / 2)} \\
\cos \frac{\theta^{c D}\left(l, n_{\lambda}\right)}{2} e^{(i / 2)(\varphi+\pi / 2)}
\end{array}\right)
\end{gathered}
$$

with the spin texture

$$
\begin{aligned}
& \theta^{c D}\left(l, n_{\lambda}\right) \\
& \quad=\arctan \left\{\frac{2 m_{h h} \beta_{h}}{\hbar^{2} R_{3}^{2 / 3}}\left[\frac{2}{3} R_{0}+\left(l^{2}-\frac{5}{4}-3 n_{\lambda}^{2}+2 \frac{n_{\lambda}^{3}}{l}\right) R_{3}\right]\right\},
\end{aligned}
$$

and the energies

$$
E_{l \sigma}=E_{a}\left(\left(l-n_{\lambda}\right)^{2}+\frac{1}{4}+\sigma \frac{\left(l-n_{\lambda}\right)}{\cos \theta^{c D}\left[\left(l-n_{\lambda}\right), n_{\lambda}\right]}\right) .
$$


We can obtain $n_{\lambda}$ from the current constraint by noting that the state $l \sigma$ carries the current

$$
\begin{aligned}
I_{l \sigma}^{\varphi}= & -\frac{e}{2 \pi}\left\{\frac{\hbar}{m a^{2}} l-\sigma \frac{\hbar}{2 m a^{2}} \cos \theta^{c D}(l)\right. \\
& \left.+\sigma \frac{\beta_{h}}{\hbar}\left(G_{1}-i G_{2}-3 G_{3}\left[l^{2}+\frac{1}{4}\right]\right) \sin \theta^{c D}(l)\right\} .
\end{aligned}
$$

We now derive analytical expressions for $n_{\lambda}$ in the weak spin-orbit coupling limit, i.e., for small $\theta^{c D}$. Subsequently, we also present numerical results for larger SOI strengths. For small angles $\theta^{c D}$, the expectation value of the current reduces to

$$
\begin{aligned}
I_{l \sigma}^{\varphi} \approx & -\frac{e}{2 \pi}\left\{\frac{\hbar}{m a^{2}} l-\sigma \frac{\hbar}{2 m a^{2}}\right. \\
& +\sigma \frac{\beta_{h}^{2}}{\hbar E_{a}^{h}}\left(G_{1}-i G_{2}-3 G_{3}\left[l^{2}+\frac{1}{4}\right]\right) \\
& \left.\times\left[\frac{2}{3} R_{0}+\left(l^{2}-\frac{5}{4}-3 n_{\lambda}^{2}+2 \frac{n_{\lambda}^{3}}{l}\right) R_{3}\right]\right\} .
\end{aligned}
$$

At zero temperature,

$$
I_{\varphi}=\sum_{\sigma= \pm 1} \sum_{-n_{r}+n_{\lambda}-\sigma / 2}^{n_{r}+n_{\lambda}-\sigma / 2} I_{l \sigma}^{\varphi}=I
$$

where $n_{r} \approx \sqrt{\epsilon_{F} / E_{a}^{h}}$ and $\epsilon_{F}$ is the Fermi energy in the absence of current. Taking $\cos \theta^{c D} \approx 1$ in the boundaries of the summation,

$$
n_{\lambda} \approx \frac{\hbar}{4 E_{a}^{h}} \frac{\pi}{e} \frac{I}{n_{r}}\left[1+3\left(\frac{\beta_{h} R_{3} n_{r}}{E_{a}}\right)^{2}\right] \approx \frac{\pi \hbar}{4 e E_{a}^{h}} \frac{I}{n_{r}},
$$

which in the limit of weak SOI does not depend on $\beta_{h}$. We find that the system is now spin polarized in the $z$ direction. With

$$
\begin{aligned}
\left\langle\sigma_{z}\right\rangle_{l \sigma} & =\left\langle\psi_{\left(l, n_{\lambda}\right) \sigma}^{c D}\left|\sigma_{z}\right| \psi_{\left(l, n_{\lambda}\right) \sigma}^{c D}\right\rangle \\
& =\sigma \cos \theta^{c D}\left(l, n_{\lambda}\right) \approx \sigma\left[1-\theta^{c D}\left(l, n_{\lambda}\right)^{2}\right],
\end{aligned}
$$

the total spin polarization is

$$
\begin{aligned}
\left\langle\sigma_{z}\right\rangle_{I}^{c D} & =\sum_{n \sigma} \sigma f_{n \sigma} \cos \theta^{c D}\left(l, n_{\lambda}\right) \\
& \approx \sum_{\sigma= \pm 1} \sum_{n=-n_{r}-n_{\lambda}+\sigma / 2}^{n_{r}-n_{\lambda}+\sigma / 2} \sigma\left\{1-\left[\theta^{c D}\left(l, n_{\lambda}\right)\right]^{2}\right) .
\end{aligned}
$$

This leads to

$$
\begin{aligned}
\left\langle\sigma_{z}\right\rangle_{I}^{c D}= & \frac{\beta_{h}^{2}}{\left(E_{a}^{h}\right)^{2} a^{6}}\left\{-4 n_{\lambda}^{3} n_{r}+64 \frac{n_{\lambda}^{6}}{n_{r}^{3}}\left(n_{\lambda}-\frac{1}{2}\right)\right. \\
& +2 n_{r} n_{\lambda}\left[4 n_{\lambda}^{2}+1+\left(n_{r}+1\right)\left(2 n_{r}+1\right)\right] \\
& \left.-\left[\frac{a^{2}}{w^{2}}-\left(\frac{5}{4}+3 n_{\lambda}^{2}\right)\right]\left(-4 n_{\lambda} n_{r}-16 \frac{n_{\lambda}^{4}}{n_{r}^{2}}\right)\right\}
\end{aligned}
$$

To the leading order in the current $n_{\lambda}$,

$$
\begin{aligned}
\left\langle\sigma_{z}\right\rangle_{I}^{c D} & \rightarrow \frac{2 \beta_{h}^{2}}{\left(E_{a}^{h}\right)^{2}} \frac{n_{r} n_{\lambda}}{a^{6}} \\
& \times\left[1+\left(n_{r}+1\right)\left(2 n_{r}+1\right)+\frac{2 a^{2}}{w^{2}}-\frac{5}{2}\right] .
\end{aligned}
$$

The total number of electrons

$$
N=\sum_{n \sigma} f_{n \sigma} \approx \sum_{\sigma= \pm 1} \sum_{-n_{r}+n_{\lambda}-\sigma / 2}^{n_{r}+n_{\lambda}-\sigma / 2} 1=4 n_{r}
$$

For $n_{r} \gg a / w$, the term proportional to $2 n_{r}^{3}$ dominates and the spin polarization simplifies to

$$
\left\langle\sigma_{z}\right\rangle_{I_{\varphi}}^{c D} \approx \frac{4 \beta_{h}^{2}}{E_{a}^{h 2}} R_{3}^{2} n_{r}^{3} n_{\lambda}=\frac{\epsilon_{F}}{E_{a}^{h}} \frac{\pi \hbar \beta_{h}^{2}}{\left(E_{a}^{h} a^{2}\right)^{3}} \frac{I}{e},
$$

while in the limit of a wide and narrow ring

$$
\left\langle\sigma_{z}\right\rangle_{I_{\varphi}}^{c D} \approx\left\langle\sigma_{z}\right\rangle_{I}^{c D} \rightarrow \frac{4 \beta_{h}^{2}}{\left(E_{a}^{h} a^{2}\right)^{2}} \frac{n_{r} n_{\lambda}}{w^{2}}=\frac{a^{2}}{w^{2}} \frac{\pi \hbar \beta_{h}^{2}}{\left(E_{a}^{h} a^{2}\right)^{3}} \frac{I}{e} .
$$

The spin polarization is in both cases proportional to the current and the squared amplitude of the SOI interaction, which is expected. The proportionality with Fermi energy when $n_{r} \gg a / w$ reflects the increasing spin texture angle $\theta^{c D}$ with energy. This implies the scaling with the squared number of particles as well as the area of the ring. In the opposite limit, we find that the spin polarization increases when tightening the laterally quantized subband because this enhances the SOI matrix elements. For realistic and currently experimentally feasible dimensions, the former approximation seems more appropriate, and thus, we focus on this limit henceforth. One can estimate the the spin polarization in this regime from Eq. (67) and the Dresselhaus coupling constant for GaAs [40] $\beta_{h}=30 \mathrm{eV} \AA^{3}$ as

$$
\left\langle\sigma_{z}\right\rangle_{I_{\varphi}}^{c D} \approx 0.2\left(\frac{\epsilon_{F}}{10 \mathrm{meV}}\right)\left(\frac{a^{2}}{\mu \mathrm{m}}\right)\left(\frac{I}{\mathrm{nA}}\right)\left(\frac{\beta_{h}}{30 \mathrm{eV \AA}^{3}}\right)^{2} .
$$

For better understanding, we can derive the equivalent effective magnetic field that would generate the same spin polarization (67) in the absence of SOI. Consider the Hamiltonian $H_{B}=p^{2} /\left(2 m_{h h}\right) \hat{1}-\Delta \sigma_{z}$, with the Zeeman energy $\Delta=\hbar e g_{h} B_{\text {eff }} /\left(4 m_{h h}\right)$, where $g_{h}$ is the gyromagnetic ratio. Clearly, such a system is spin polarized and in the limit of $\Delta / \epsilon_{F} \ll 1$,

$$
\left\langle\sigma_{z}\right\rangle_{Z} \approx \frac{\hbar}{2} \frac{e g_{h} B_{\mathrm{eff}}}{m_{h h}} \frac{1}{\sqrt{E_{a}^{h} \epsilon_{F}}}
$$

The $\epsilon_{F}^{-1 / 2}$ dependence reflects the 1D density of states that decreases with energy. Comparison of Eqs. (70) and (67) gives an equivalent effective field of

$$
B_{\mathrm{eff}}=\frac{32 \pi}{\sqrt{2}} \frac{m_{h h}^{9 / 2} a}{e \hbar^{7} g_{h}} \epsilon_{F}^{3 / 2} \beta_{h}^{2} \frac{I}{e},
$$

where we assume the $g$ factor $g_{h}=-0.5$ [41]. Inserting the parameters

$$
B_{\text {eff }}=1.3\left(\frac{\epsilon_{F}}{10 \mathrm{meV}}\right)^{3 / 2} \frac{a}{1 \mu \mathrm{m}} \frac{I}{\mathrm{nA}}\left(\frac{\beta_{h}}{30 \mathrm{eVA}^{3}}\right)^{2} \mathrm{mT},
$$

we find that light-induced current of the order of $10 \mathrm{nA}$ (see Appendix) generates the effective field of roughly $10 \mathrm{mT}$. 

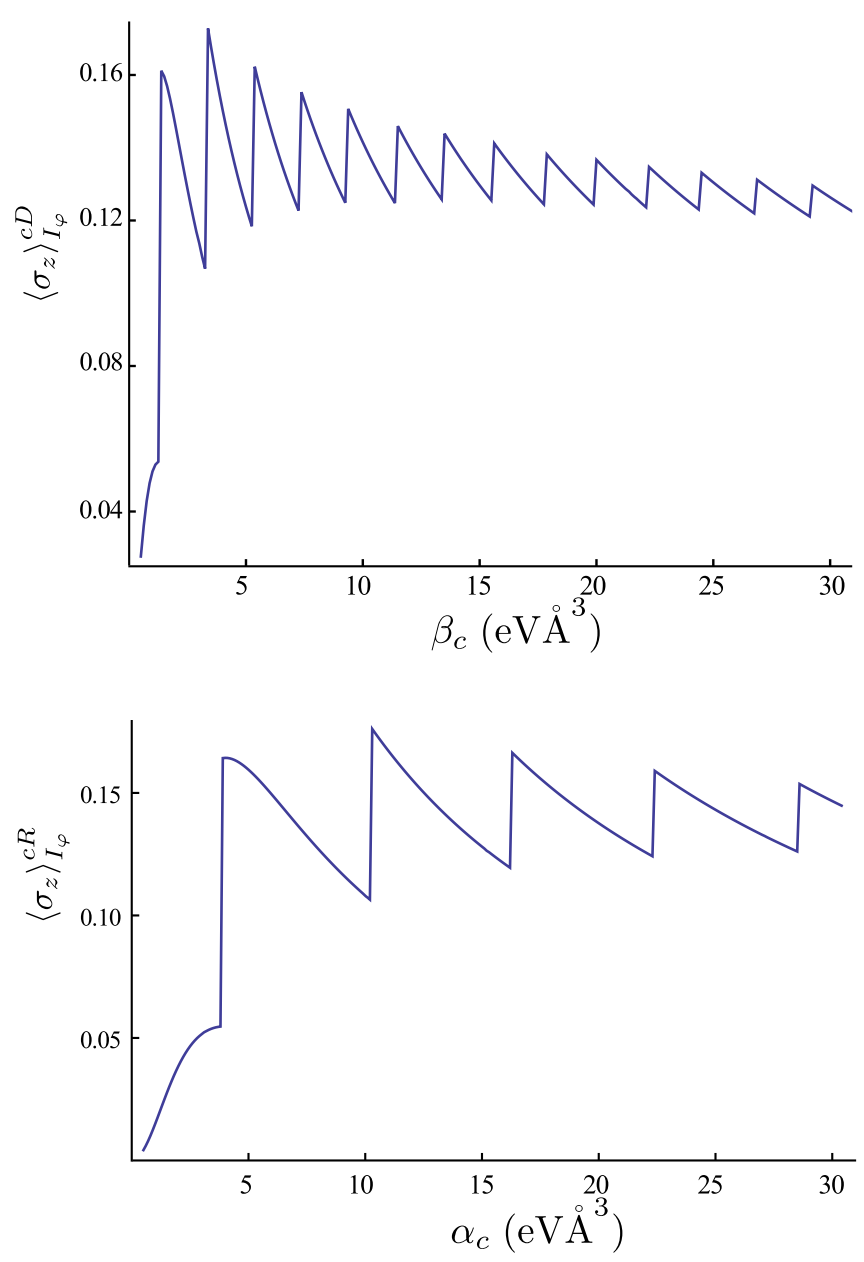

FIG. 3. (Color online) The current-induced spin polarization of heavy holes in a quantum ring subject to the cubic Dresselhaus (Rashba) SOI, plotted in the upper (lower) panel. The plots are shown as a function of the SOI parameters and $m_{h h}=0.45 m_{0}, N=1144$, which in the absence of current is equivalent to $\epsilon_{F}=10 \mathrm{meV}$, a radius of $a=1 \mu \mathrm{m}$, and width of $w=50 \mathrm{~nm}$. Here, we assumed a current of $I=35 \mathrm{nA}$, which is equivalent to circularly polarized light with the frequency of $\omega=2 \times 10^{14} \mathrm{~s}^{-1}$, and the electric field amplitude of $\left|E_{0}\right|=\sqrt{60} \times 10^{7} \mathrm{Vm}^{-1}$ (see Appendix).

Keeping in mind that the current is quantized in steps as a function of the system parameters as discussed above, the spin polarization computed numerically increases linearly with the current level up to $I=100 \mathrm{nA}$, in agreement with the analytic result. The deviations from the perturbation theory are quite large for the spin-orbit interaction parameter for GaAs used above. The nonperturbative numerical results for the spin polarization are plotted as a function of SOI strengths for constant electron numbers in Fig. 3. We observe that at small $\beta_{h}$ the spin polarization increases quadratically with SOI as found in the weak SOI limit above but saturates at higher values. We also observe a sawtoothlike behavior on top of this trend that is caused by a repopulation of states: SOI induces a spin polarization when the current bias shifts the occupation numbers around the Fermi level. For small but increasing $\beta_{h}$, we expect an increasing spin polarization with SOI at constant current since the state dependence of the spin
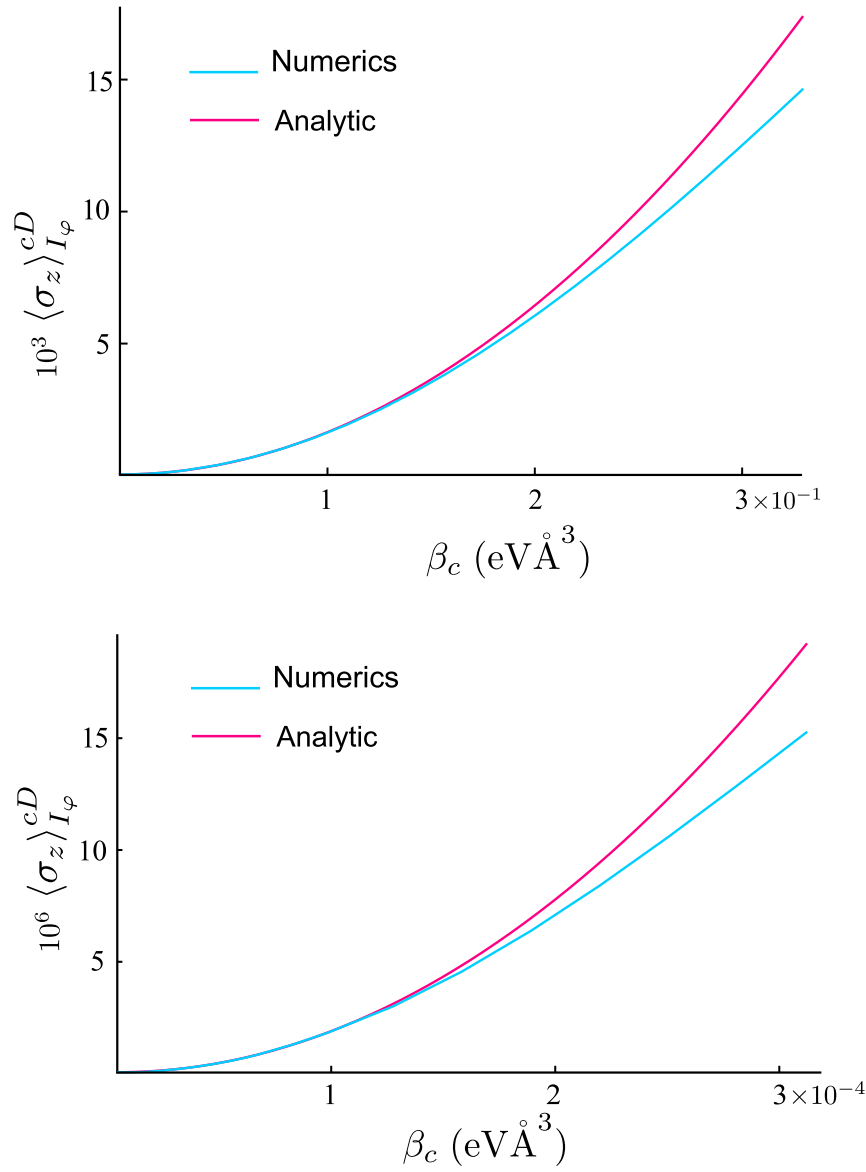

FIG. 4. (Color online) Comparison of analytical and numerical results in the regime of the small Dresselhaus SOI for holes orbiting in a ring. Upper panel: $n_{r}^{2} \gg a^{2} / w^{2}$. The parameters are the same as in Fig. 3 in this limit. Lower panel: limit of $n_{r}^{2} \ll a^{2} / w^{2}$ with $w=1$ $\mathrm{nm}$. The other parameters are the same as in Fig. 3.

texture increases. At large $\beta_{h}$, on the other hand, the angle of the spin with respect to the $z$ axis $\theta^{c D}\left(l, n_{\lambda}\right)$ can be large, corresponding to smaller values of the $z$ component of the spin. Thus, by further increasing $\beta_{h}$, the overall polarization saturates and even slightly decreases. The jumps reflect level crossings with increasing $\beta_{h}$. In these calculations, the number of electrons is kept constant (the Fermi energy oscillates). At such a discontinuity, an electron vacates a high angular momentum state in favor of a smaller one, which reduces the total spin polarization. In the regime of small SOI, the numerical and analytical results for the current-induced spin polarization agree well (see Fig. 4).

\section{Holes with the Rashba SOI}

The Hamiltonian of holes in the presence of the Rashba SOI is [38]

$$
\begin{aligned}
H_{0}^{c R}= & -\frac{\hbar^{2}}{2 m_{h h} a^{2}} \partial_{\varphi}^{2} \\
& +i \alpha_{h} e^{3 i \varphi}\left(F_{0}+F_{1} \partial_{\varphi}+F_{2} \partial_{\varphi}^{2}+F_{3} \partial_{\varphi}^{3}\right) \sigma_{-} \\
& -i \alpha_{h} e^{-3 i \varphi}\left(F_{0}-F_{1} \partial_{\varphi}+F_{2} \partial_{\varphi}^{2}-F_{3} \partial_{\varphi}^{3}\right) \sigma_{+},
\end{aligned}
$$


where $F_{0}=i\left(R_{0}-3 R_{1}+3 R_{2}\right), F_{1}=-3 R_{1}+9 R_{2}-8 R_{3}$, $F_{2}=i\left(-3 R_{2}+6 R_{3}\right)$, and $F_{3}=R_{3}$. $R_{i}$ 's depend on the radial confinement and are defined in Sec. IV C.

The current operator in spin space is

$$
\begin{aligned}
\frac{\hat{I}_{l}^{\varphi}}{e}= & 2 E_{a}^{h}\left(l_{-}^{\prime} \sigma_{1}+l_{+}^{\prime} \sigma_{2}\right) \\
& -\frac{\alpha_{h}}{\hbar}\left(F_{1}+2 i F_{2} l_{-}^{\prime}+3 F_{3} l_{-}^{\prime 2}\right) \sigma_{-} \\
& -\frac{\alpha_{h}}{\hbar}\left(F_{1}-2 i F_{2} l_{+}^{\prime}+3 F_{3} l_{+}^{\prime 2}\right) \sigma_{+},
\end{aligned}
$$

where $l_{+}^{\prime}=l+1$ and $l_{-}^{\prime}=l-2$. Thus, the same procedure as before leads to the Hamiltonian carrying a ground-state current. In spin space,

$$
\begin{aligned}
H^{c R}= & \left.H_{0}^{c R}\left(l-n_{\lambda}\right)\right|_{\bar{F}_{1} \rightarrow F_{1}, \bar{F}_{0} \rightarrow F_{0}} \\
& -2 \alpha_{h} e^{i \varphi} F_{3} n_{\lambda}^{3} \sigma_{-}+2 \alpha_{h} F_{3} n_{\lambda}^{3} e^{-i \varphi} \sigma_{+},
\end{aligned}
$$

where $\bar{F}_{1}=F_{1}+3 F_{3} n_{\lambda}^{2}$ and $\bar{F}_{0}=F_{0}-F_{2} n_{\lambda}^{2}$, and we disregard a constant shift of $-\hbar^{2} n_{\lambda}^{2} /\left(2 m_{h h} a^{2}\right)$ in the Hamiltonian. Eigenstates are now

$$
\begin{gathered}
\psi_{\left(l, n_{\lambda}\right),+}^{c R}=e^{i l \varphi}\left(\begin{array}{c}
\cos \frac{\theta^{c R}\left(l, n_{\lambda}\right)}{2} e^{-(3 i / 2)(\varphi)} \\
\sin \frac{\theta^{c R}\left(l, n_{\lambda}\right)}{2} e^{(3 i / 2)(\varphi)}
\end{array}\right) ; \\
\psi_{\left(l, n_{\lambda}\right),-}^{c R}=e^{i l \varphi}\left(\begin{array}{c}
-\sin \frac{\theta^{c R}\left(l, n_{\lambda}\right)}{2} e^{-(3 i / 2)(\varphi)} \\
\cos \frac{\theta^{c R}\left(l, n_{\lambda}\right)}{2} e^{(3 i / 2)(\varphi)}
\end{array}\right),
\end{gathered}
$$

where the texture angle $\theta^{c R}\left(l, n_{\lambda}\right)$ is

$$
\begin{aligned}
& \theta^{c R}\left(l, n_{\lambda}\right) \\
& \quad=\tan ^{-1}\left\{\tilde{\alpha}_{h}\left[\frac{2}{3} R_{0}+\left(\frac{13}{12}-\frac{1}{3} l^{2}+n_{\lambda}^{2}-\frac{2}{3} \frac{n_{\lambda}^{3}}{l}\right) R_{3}\right]\right\},
\end{aligned}
$$

with $\tilde{\alpha}_{h}=2 m_{h h} \alpha_{h} /\left(\hbar^{2} R_{3}^{2 / 3}\right)$ and the energies

$$
E_{k, \sigma}=E_{a}\left(\left(l-n_{\lambda}\right)^{2}+\frac{1}{4}+\sigma \frac{\left(l-n_{\lambda}\right)}{\cos \left\{\theta^{c R}\left[\left(l-n_{\lambda}\right), n_{\lambda}\right]\right\}}\right) .
$$

For small $\theta^{c R}$ we find, as above,

$$
n_{\lambda} \approx-\frac{\hbar}{4 E_{a}^{h}} \frac{\pi}{e} \frac{I}{n_{r}}
$$

and a spin polarization in the $z$ direction:

$$
\left\langle\sigma_{z}\right\rangle_{I_{\varphi}}^{c R} \approx-\frac{\epsilon_{F}}{E_{a}^{h}} \frac{\pi \hbar \alpha_{h}^{2}}{9\left(E_{a}^{h} a^{2}\right)^{3}} \frac{I}{e},
$$

very similar to the Dresselhaus limit, but with a prefactor $\frac{1}{9}$. Therefore, the above discussions for the small SOI limit hold for the cubic Rasba Hamiltonian as well. In the lower part of Fig. 3, we plotted numerical results for larger values of $\alpha_{h}$. The values of the SO coupling used in this figure can be experimentally achieved (e.g., Ref. [26]) by external gate voltage.

\section{CONCLUSIONS}

IFE allows in principle ultrafast and nondissipative actuation and eventual switching of magnetization. We investigated the impact of the SOI on this nonabsorbing "opto-spin" phenomena. We provided a proof of principle for a mechanism that is based on the current-induced generation of a spin polarization that would generate torques in a magnetic sample. The current bias can be generated by the Lagrange multiplier method inspired by current-density functional theory. For electrons moving in quantum rings in the presence of the Rashba and the Dresselhaus SOIs, the effect vanishes. It becomes nonzero only when the Kramers' degeneracy is broken by an exchange potential or applied magnetic field, but the effects are still small [35]. On the other hand, holes in a ring with cubic Dresselhaus and Rashba SOI display spin polarization under current bias. This polarization is a competition between two effects. On one hand, with increasing SOI, the band splitting increases, which amplifies the magnitude of the polarization. Simultaneously, however, the $z$ component of the spin of electrons with energies near to the Fermi level decreases, and therefore the net polarization decreases. These two might enhance the effect rather than cancel each other when the spin texture would push the spin out of the plane. This can be achieved in a ring with an asymmetric potential in the radial direction, such as a thin slice of GaAs $\mid p$ doped GaAlAs core/shell nanowire. The second Rashba SOI would pull the spin toward the $z$ direction and lead to monotonic increase of current-induced spin polarization with SOI.

Induced polarization in the $z$ direction, calculated in this paper, could be either parallel or antiparallel to the $z$ axis depending on the direction of the current. This is consistent with the IFE in which the effective magnetic field changes sign with the helicity of light. Here, we focused on the spin polarization induced by current in a material which is nonmagnetic. This spin polarization can be measured directly by pump and Kerr rotation probe measurements. GaMnAs in the ferromagnetic state is a hole conductor. Here, the current-induced spin polarization would induce torques on the magnetic order parameter, eventually causing magnetization switching. The spin-dependent dynamic Stark effect also induces torques by circularly polarized light [14]. The two processes are independent and should be added. They can be distinguished by tuning the light frequency close to the energy gap, where the dynamic Stark effect is resonantly enhanced.

The currents generated by nonresonant light are persistent, analogous to the diamagnetic currents in conducting rings induced by dc magnetic fields [23]. While this issue has not been central to our study, our results imply that the spin-orbit interaction can induce large paramagnetic corrections to the diamagnetic response. Cantilever-based torsional magnetometers with integrated mesoscopic rings allow very sensitive measurements of magnetic susceptibilities [42]. We suggest that quantum ring arrays made from 2 DHGs would be interesting subjects for such experiments.

\section{ACKNOWLEDGMENTS}

This work was supported by FOM (Stichting voor Fundamenteel Onderzoek der Materie), by the ICC-IMR, by DFG Priority Programme 1538 "Spin-Caloric Transport" (BA 
2954/1), and by JSPS Grants-in-Aid for Scientific Research Grants No. 25247056 and No. 25220910.

\section{APPENDIX: LIGHT INDUCED CURRENTS}

Here, we show how to use the collisionless plasma model by Hertel [19] to obtain the light-induced current in a quantum ring. This model can be used for the present system in the high-frequency limit, in which the path an electron traverses under a half-cycle of the oscillating light electric field is much smaller than the characteristic length scales such as the finite radial thickness or the spin-orbit precession length.

Hertel finds a circular current as a result of the circularly polarized light in the form of

$$
\mathbf{j}_{\varphi}=-\frac{i}{4 e\langle n\rangle \omega} \nabla \times\left[\sigma^{*} \mathbf{E}^{*} \times \sigma \mathbf{E}\right],
$$

where $\mathbf{E}$ is the electric component of the light field and

$$
\sigma=\frac{i\langle n\rangle e^{2}}{m \omega}
$$

is the high-frequency conductivity of a collisionless plasma, with $\langle n\rangle$ the volume density of the electrons and $\omega$ the light frequency. For circularly polarized light with helicity $\Lambda= \pm$

$$
\mathbf{E} \times \mathbf{E}^{*}=\Lambda i|E|^{2} \mathbf{e}_{z} .
$$

Thus,

$$
\begin{aligned}
\mathbf{j}_{\varphi}(r) & =\Lambda \frac{\langle n\rangle e^{3}}{4 m^{2} \omega^{3}} \nabla \times\left(|E(r)|^{2} \mathbf{e}_{z}\right) \\
& =-\Lambda \frac{\langle n\rangle e^{3}}{4 m^{2} \omega^{3}}\left(\frac{\partial|E(r)|^{2}}{\partial r}\right) \mathbf{e}_{\varphi} .
\end{aligned}
$$

Since this result does not depend on the $z$ coordinate, it holds for a 2DEG and normally incident light.

In a ring we can project the current to one dimension by writing the current density

$$
\mathbf{j}_{\varphi}=j_{\mathrm{ext}}^{1 \mathrm{D}} \mathbf{e}_{\varphi},
$$

where

$$
\begin{aligned}
j_{\mathrm{ext}}^{1 \mathrm{D}} & =\left\langle R_{0}(r) Z_{0}(z)\left|j_{\mathrm{ext}}(r)\right| Z_{0}(z) R_{0}(r)\right\rangle \\
& =-\Lambda \frac{\langle n\rangle e^{3}}{4 m^{2} \omega^{3}} \int d r\left|R_{0}(r)\right|^{2} \frac{\partial|E(r)|^{2}}{\partial r} .
\end{aligned}
$$

We consider a laser spot with Gaussian spatial distribution

$$
\begin{gathered}
\mathbf{E}(r)=\left(\mathbf{e}_{x}+\Lambda i \mathbf{e}_{y}\right) E_{0} \exp \left(-\frac{\gamma r^{2}}{2}\right), \\
|E|^{2}=E_{0}^{2} \exp \left(-\gamma r^{2}\right),
\end{gathered}
$$

where $E_{0}$ is the maximum value of the electric field in the spot center. Thus, the total current in the ring with radius $a$ then becomes

$$
I=\iint d z d r j_{\mathrm{ext}}^{1 \mathrm{D}}=\Lambda \gamma E_{0}^{2} \exp \left(-a^{2} \gamma\right) \frac{N e^{3}}{4 \pi m^{2} \omega^{3}},
$$

where we used $\int d r \int d z\langle n\rangle=N /(2 \pi a)$, the linear density of a ring with $N$ electrons. The above current has dimension of ampere. The result is also valid for the holes (with modified mass and opposite current direction). The light intensity reads as, in terms of the electric field,

$$
\text { Intensity }=\frac{c n^{\prime} \epsilon}{2}|E|^{2},
$$

where $c$ is the velocity of light in vacuum, $\epsilon$ is the dielectric constant, and $n^{\prime}$ the index of refraction. We estimate the current by assuming $\epsilon \approx 10 \epsilon_{0}, n^{\prime} \approx 3$. At a typical laser intensity of $10^{13} \mathrm{Wm}^{-2}$ or, equivalently, $\left|E_{0}\right|^{2} \approx 3 \times 10^{15} \mathrm{~V}^{2} \mathrm{~m}^{-2}$, and wavelength/frequency $\lambda^{\prime}=12 \pi \mu \mathrm{m} / \omega=2 \times 10^{14} \mathrm{~s}^{-1}$ used in all-optical switching [43], we find for the current in a 2DHG ring

$$
\begin{aligned}
|I|= & 16 \mathrm{nA} \frac{E_{0}^{2}}{3 \times 10^{15} \mathrm{~V}^{2} \mathrm{~m}^{-2}} \frac{\gamma \exp \left(-a^{2} \gamma\right)}{10^{12} \exp (-1) \mathrm{m}^{-2}} \frac{N}{1000} \\
& \times\left(\frac{0.45 m_{0}}{m}\right)^{2}\left(\frac{2 \times 10^{14} \mathrm{~s}^{-1}}{\omega}\right)^{3} .
\end{aligned}
$$

[1] L. P. Pitaevskii, Zh. Eksp. Teor. Fiz. 39, 1450 (1960) [Sov. Phys.-JETP 12, 1008 (1961)].

[2] J. P. van der Ziel, P. S. Pershan, and L. D. Malmstrom, Phys. Rev. Lett. 15, 190 (1965).

[3] P. S. Pershan, J. P. van der Ziel, and L. D. Malmstrom, Phys. Rev. 143, 574 (1966).

[4] D. Popova, A. Bringer, and S. Blügel, Phys. Rev. B 84, 214421 (2011).

[5] D. Popova, A. Bringer, and S. Blügel, Phys. Rev. B 85, 094419 (2012).

[6] A. V. Kimel, A. Kirilyuk, P. A. Usachev, R. V. Pisarev, A. M. Balbashov, and Th. Rasing, Nature (London) 435, 655 (2005).
[7] A. Kirilyuk, A. V. Kimel, and T. Rasing, Rev. Mod. Phys. 82, 2731 (2010).

[8] C. D. Stanciu, F. Hansteen, A. V. Kimel, A. Kirilyuk, A. Tsukamoto, A. Itoh, and Th. Rasing, Phys. Rev. Lett. 99, 047601 (2007).

[9] K. Vahaplar, A. M. Kalashnikova, A. V. Kimel, S. Gerlach, D. Hinzke, U. Nowak, R. Chantrell, A. Tsukamoto, A. Itoh, A. Kirilyuk, and Th. Rasing, Phys. Rev. B 85, 104402 (2012).

[10] N. Kazantseva, D. Hinzke, U. Nowak, R. W. Chantrell, U. Atxitia, and O. Chubykalo-Fesenko, Phys. Rev. B 77, 184428 (2008).

[11] R. V. Mikhaylovskiy, E. Hendry, and V. V. Kruglyak, Phys. Rev. B 86, 100405 (2012). 
[12] M. Battiato, G. Barbalinardo, and P. M. Oppeneer, Phys. Rev. B 89, 014413 (2014).

[13] K. Taguchi and G. Tatara, Phys. Rev. B 84, 174433 (2011).

[14] A. Qaiumzadeh, G. E. W. Bauer, and A. Brataas, Phys. Rev. B 88, 064416 (2013).

[15] V. I. Karpman and A. G. Shagalov, J. Plasma. Phys. 27, 215 (1982).

[16] V. Tsytovich, Commun. Plasma Phys. Cont. Fusion 4, 81 (1978).

[17] S. A. Belkov and V. N. Tsytovich, Zh. Eksp. Teor. Fiz. 76, 1293 (1979) [Sov. Phys.-JETP. 49, 656 (1979)].

[18] M. Kono, M. M. Skoric, and D. Ter Haar, J. Plasma Phys. 26, 123 (1981).

[19] R. Hertel, J. Magn. Magn. Mater. 303, L1 (2006).

[20] T. Yoshino, J. Magn. Magn. Mater. 323, 2531 (2011).

[21] A. Manchon and S. Zhang, Phys. Rev. B 78, 212405 (2008).

[22] A. Manchon and S. Zhang, Phys. Rev. B 79, 094422 (2009).

[23] M. Büttiker, Y. Imry, and R. Landauer, Phys. Lett. A 96, 365 (1983).

[24] O. V. Kibis, Phys. Rev. Lett. 107, 106802 (2011).

[25] J. Inoue, T. Kato, G. E. W. Bauer, and L. W. Molenkamp, Semicond. Sci. Technol. 24, 064003 (2009).

[26] X. C. Zhang, A. Pfeuffer-Jeschke, K. Ortner, V. Hock, H. Buhmann, C. R. Becker, and G. Landwehr, Phys. Rev. B 63, 245305 (2001).

[27] R. Winkler, Phys. Rev. B 62, 4245 (2000).

[28] G. Vignale, Phys. Rev. B 70, 201102(R) (2004).

[29] F. E. Meijer, A. F. Morpurgo, and T. M. Klapwijk, Phys. Rev. B 66, 033107 (2002).
[30] F. J. Ohkawa and Y. Uemura, J. Phys. Soc. Jpn. 37, 1325 (1974).

[31] Y. A. Bychkov and E. I. Rashba, J. Phys. C: Solid State Phys. 17, 6039 (1984).

[32] G. Dresselhaus, Phys. Rev. 100, 580 (1955).

[33] R. Winkler, H. Noh, E. Tutuc, and M. Shayegan, Phys. Rev. B 65, 155303 (2002).

[34] D. V. Bulaev and D. Loss, Phys. Rev. Lett. 95, 076805 (2005).

[35] F. Joibari (unpublished).

[36] M. Edelstein, Solid State Commun. 73, 233 (1990).

[37] B. Berche, C. Chatelain, and E. Medina, Eur. J. Phys. 31, 1267 (2010).

[38] D. Stepanenko, M. Lee, G. Burkard, and D. Loss, Phys. Rev. B 79, 235301 (2009).

[39] A. A. Kovalev, M. F. Borunda, T. Jungwirth, L. W. Molenkamp, and J. Sinova, Phys. Rev. B 76, 125307 (2007).

[40] J. B. Miller, D. M. Zumbuhl, C. M. Marcus, Y. B. Lyanda-Geller, D. Goldhaber-Gordon, K. Campman, and A. C. Gossard, Phys. Rev. Lett. 90, 076807 (2003).

[41] M. Rubinstein, A. Hanbicki, P. Lubitz, M. Osofsky, J. J. Krebs, and B. Jonker, J. Magn. Magn. Mater. 250, 164 (2002).

[42] A. C. B. Jayich, W. E. Shanks, B. Peaudecerf, E. Ginossar, F. von Oppen, L. Glazman, and J. G. E. Harris, Science 326, 272 (2009).

[43] K. Vahaplar, A. M. Kalashnikova, A. V. Kimel, D. Hinzke, U. Nowak, R. Chantrell, A. Tsukamoto, A. Itoh, A. Kirilyuk, and Th. Rasing, Phys. Rev. Lett. 103, 117201 (2009). 